\section{Diastereoselective Pyrrolidine Synthesis Using a Sulfinamide Annulating Agent}

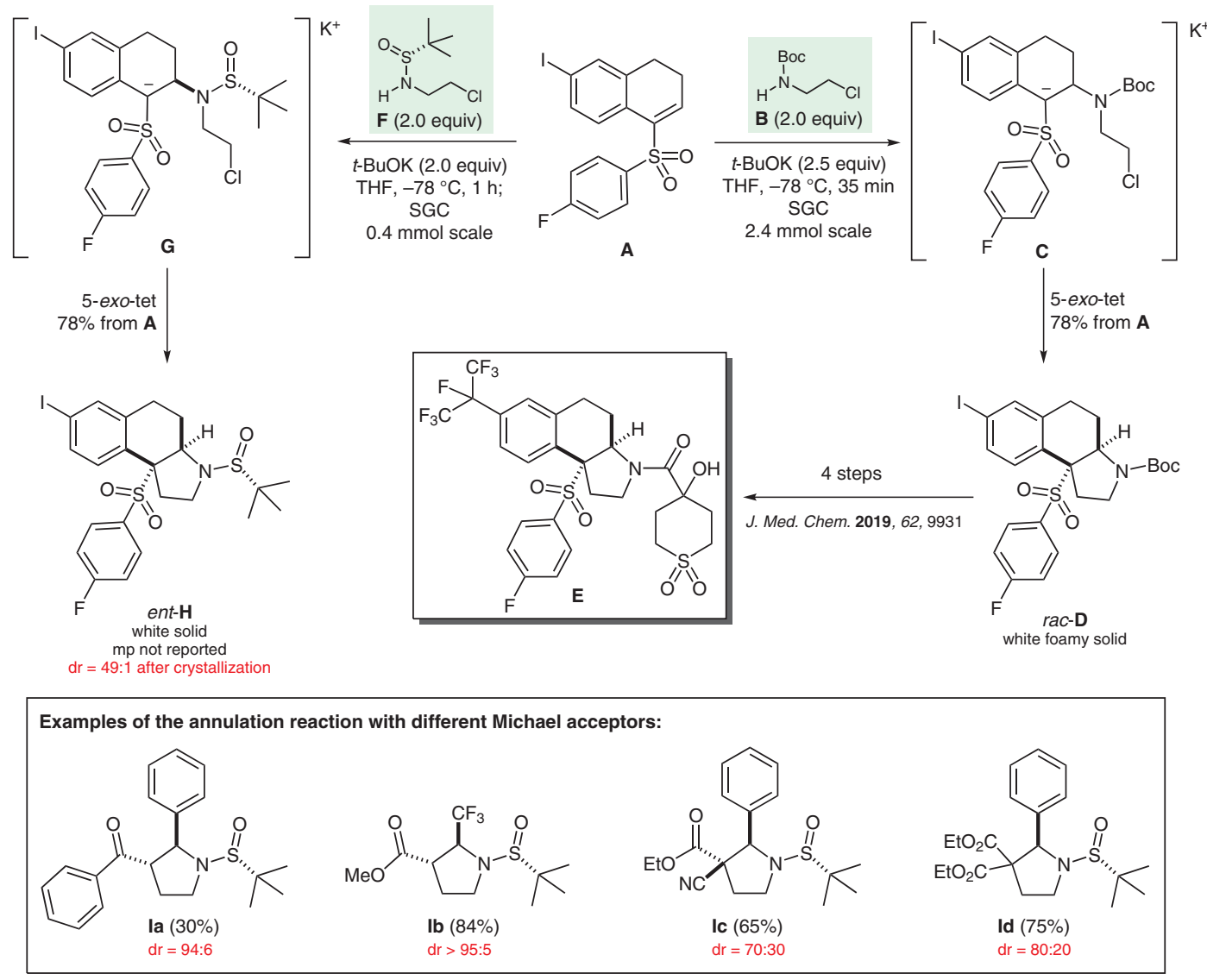

Category

Synthesis of Natural

Products and

Potential Drugs

\section{Key words}

ROR $\gamma \mathrm{t}$ inverse agonist

annulation

asymmetric aza-

Michael reaction

pyrrolidine ring

formation

5-exo-tet cyclization
Significance: A recent synthesis of the ROR $\gamma \mathrm{t}$ inverse agonist $\mathbf{E}$ (J. Med. Chem. 2019, 63, 9931) featured an aza-Michael addition of metallated carbamate $\mathbf{B}$ to Michael acceptor $\mathbf{A}$ followed by cyclization of adduct $\mathbf{C}$ to give pyrrolidine rac-D as a single diastereoisomer in $75 \%$ yield. The conversion of rac-D into the target molecule $\mathbf{E}$ then required four steps including an SFC separation of enantiomers. An asymmetric variant of the pyrrolidine annulation has now been developed that obviates the need for chromatographic resolution. Thus, the diastereoselective aza-Michael addition of chiral sulfinamide $\mathbf{F}$ to sulfone $\mathbf{A}$ afforded adduct $\mathbf{G}$ which then cyclized to give pyrrolidine ent-H in $75 \%$ yield and $\mathrm{dr}=49: 1$.
Comment: The novel and versatile annulating reagent $\mathbf{F}$ is crystalline and stable at room temperature for more than one year. Moreover, the sulfinamide group renders the corresponding products crystalline. The annulation reaction possesses a wide scope, as a variety of singly and doubly activated Michael acceptors provide the corresponding pyrrolidines in moderate to high yields with excellent diastereoselectivities (24 examples). 